\title{
An impact protective structure with bistable links
}

\author{
A. Cherkaev ${ }^{\text {a }}$, S. Leelavanichkul ${ }^{\text {b }}$ \\ a Department of Mathematics University of Utah \\ ${ }^{\mathrm{b}}$ Department of Mechanical Engineering University of Utah
}

\begin{abstract}
This paper discusses a design concept of a protective structure with a special morphology that improves the impact resistance capability. We present a "bistable" structure concept: The bistable structure is designed so that the stress-strain relation is not unique. When the strain reaches a threshold, that part of the structure yields and subsequently fails. The remaining parts then carry the loads and maintain the structural integrity. The redistribution of the loads allows more energy of the collision to be absorbed.

We describe the response of bistable structures in macro- and microscale, introduce a mesoscale damage tensor that measures the degree of damage in a small region, and numerically investigate a problem of projectile impacting a protective net. This paper introduces several effectiveness criteria of the protective bistable structure, and compares the structural responses of the bistable and conventional structure. The bistable structure dissipates more energy by creating a partial damage in a large area. Damage is understood as a process of irreversible phase transition from the initial to disintegrated state. Damage of the bistable structure is more stable; the cracks do not develop due to yielding of structural elements and damage delocalization.
\end{abstract}

Key words: protective structures, fracture, damage mechanics, bistable, waiting elements

\section{Introduction}

An unstructured material can absorb energy until it melts. However, a tiny portion of this energy yields to a construction's disintegration due to instabilities of the damage propagation that leads to energy concentration and failure. Appearance of concentrated damage zones, such as cracks or delaminating, destroys the construction, while the remains of the disintegrated construction still is able absorb a lot of energy.

\footnotetext{
* Corresponding author.

Email addresses: cherk@math.utah.edu (A. Cherkaev), sleelava@eng.utah.edu (S. Leelavanichkul).
} 
We investigate a propagation of structural damage, and present models of a protective structure with improved characteristics. The designed structures must sustain a sudden impact: they absorb the energy while maintaining their structural integrity [1]. Commonly used protective devices include car bumpers, helmets, tempered glass, climbing "screamer," and woven baskets of hot balloons. These very different constructions are all designed to be damaged or destroyed in a collision saving the protected object - main vehicle or its passengers. In a collision, a properly designed bumper will be badly damaged, thereby absorbing the energy of impact, and saving the vehicle and its passengers. A strong and stiff bumper that stays undamaged while the car is ruined does not fit its purpose. Likewise, a fragile bumper does not fit because it is easily destroyed and does not absorb enough energy to protect the vehicle. The optimally designed structure realizes the known recommendation of defensive driving instructions "Choose to hit something that will give way (such as bushes or shrubs) rather than something hard." The principle of design of protective structure is in contrast with the conventional optimal design of constructions of maximal stiffness, which should stay undamaged under a given static load.

Since damage to personnel or equipment is minimized by maximizing the energy absorbed by the protective structure, these devices are most effective when they are completely damaged or destroyed everywhere. Naturally, a concentrated impact load tends to produce concentrated zones of damage, which is contrary to the goal of maximizing damage throughout the structure. The design concept presented here leads to a special construction that creates an optimal distribution of damage throughout the structure. To spatially distribute damage, a first-stage sacrificial microstructure is used to radiate a large portion of the initial impact energy away from the impact point. After the sacrificial structure breaks, a backup "waiting element" structure provides continued resistance to catastrophic localized failure. Much like organic super-resistive structures, a waiting element structure provides a secondary resistance that is not present in conventional protective devices.

The total fracture energy is an important factor when determining the capability of a structure to withstand a dynamical loading. In most structures, however, the energy density is uneven. Localized yielding occurs at a particular location while the total energy associated with fracture in the structure is relatively small. To increase the total fracture energy, the concept of a bistability has been introduced [2]. In bistable structures, yielding is produced in a larger volume, thus increasing the total fracture energy. A bistable structure is designed with localized redundant load paths. When one load path yields and subsequently fails, the remaining load path can carry the load and retain structural integrity. A bistable structure may be developed by forming a chain or lattice of bistable structural links [3-5]. Multiple regions of such structure exhibit yielding prior to ultimate failure. Thus, the bistable structures allow for the delocalization of yielding in a structure. In this paper, we emphasize their design principles via the assembly of "sacrificial" and "waiting" elements, and advantages in comparison to conventional structures.

\section{Elements of an impact resistive bistable structure}

In all unstructured material, stress rate decreases when strain increases, due to development of imperfection, micro-cracks opening, dislocation concentration, and similar 


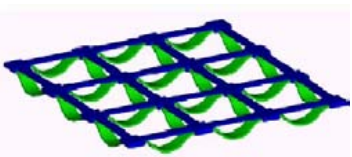

(a)

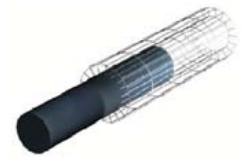

(b)

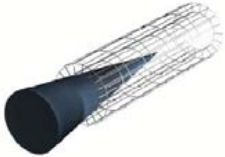

(c)

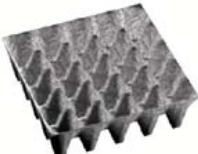

(d)

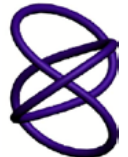

(e)

Fig. 1. Structures that absorb energy and become partially damaged, but maintain structural integrity. (a) Lattice with redundant load paths. (b) Tube and cylinder. (c) Tube and cone. (d) Multilayer structure.

(e) Structure with helices that mimics protein.
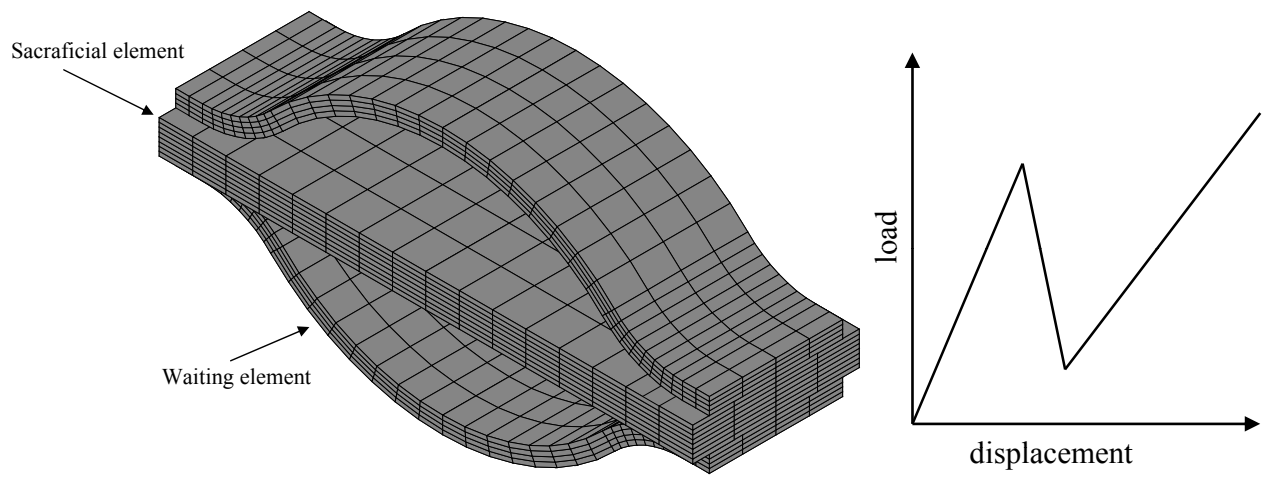

Fig. 2. A bistable link assembly and its force-displacement response

mechanisms. This phenomenon leads to stress concentration and instabilities that eventually destroy the structure. To increase the resistance capability, we suggest structures that become stiffer and stronger when damaged, thanks to their morphology. Such structures distribute the stress more evenly and are stable even when they are partially destroyed (Fig. 1). These structures can experience multiple inner breakages and they can sustain large deformations without destruction, a wave transports the damage away from the impacted region $[4,5]$.

In two-dimensional lattice, strong elastic waves and waves of damage propagate throughout the construction, reflecting from its boundaries and interfering with each other. This process leads to a high concentration of stresses that damage the construction.

\subsection{Impact-resistant bistable structure}

A desired distribution of a moderate damage, initiation and redirection of damage waves can be effectively achieved by using bistable structures. Bistable structure is an assembly of roughly parallel two brittle-elastic or elastic-plastic rods [6], one of which is longer than the other, see Fig. 2. When the shorter (sacrificial) element fails, the load is assumed by the second (waiting) element that was initially inactive. Bistable links lead to large but stable pseudo-plastic strains and thus increase the resistivity. Notice the difference between the plastic response and the one shown in Fig. 2. For large deformations, the bistable link regains stability while the plastic link would remain unstable.

Because of these local instabilities, the multiscale bistable structures excite intensive waves of partial damage and relaxes the structure by allowing for large local deforma- 


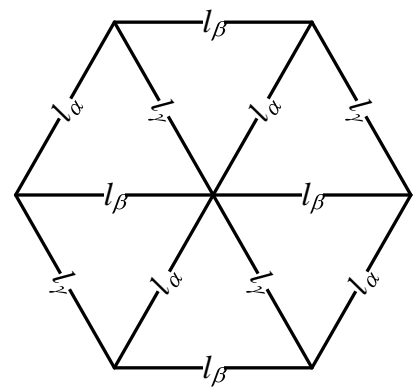

(a)

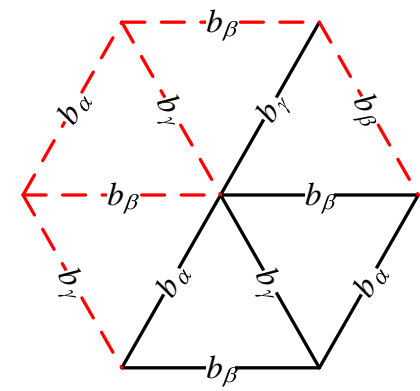

(b)

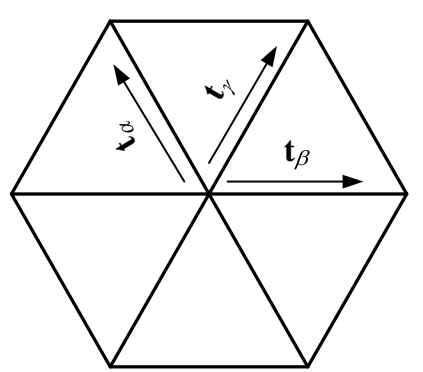

(c)

Fig. 3. (a) The lattice consists of three families of parallel links $\alpha, \beta$, and $\gamma$. (b) Example of lattice with damaged links (dashed lines). In this illustration, $b_{\alpha}=1, b_{\beta}=2$, and $b_{\gamma}=3$. (c) Tangent vectors to the links' families.

tions of the damaged links. The bistable cellular structures (lattices) containing waiting elements naturally excite and transmit waves of damage, acting as a domino train. The structure with bistable links transforms the energy of an impact into fast traveling waves that distribute energy throughout a larger area where it dissipates.

\subsection{Criteria of damage and tensor of damage}

In a chain, it is natural to count the number of partially damaged links to access the absorbed energy. Any completely broken link means that the whole chain is destroyed. To the contrary, the triangular lattice can still resist projectiles even if some of the links are completely broken. We use two basic criteria that compare the state of the structure before and after the collision:

(i) The percentage of partially damaged links

(ii) The percentage of destroyed links

The first number shows how effectively the damage is spread, and the second one shows how badly the structure is damaged. Ideally, we wish to have a structure in which all links are partially damaged, but none is completely destroyed.

The number of destroyed links is a rough quality criterion. It ignores a significant factor - the positions of the destroyed links. while the effect of the damage depends on the distribution and orientation of the broken links. To describe various configurations of broken links, we introduce a damage tensor that can distinguish partial damage depending on which links are broken. The consideration is similar for the sacrificial and the waiting elements.

The lattice consists of three families of parallel links $\alpha, \beta$, and $\gamma$ see Fig. 3a. The angle between the tangent vectors to the links' families is $60^{\circ}$. They are characterized by tangent vectors $\mathbf{t}_{\alpha}, \mathbf{t}_{\beta}, \mathbf{t}_{\gamma}$, Figure $3 \mathrm{c}$;

$$
\mathbf{t}_{\alpha} \cdot \mathbf{t}_{\beta}=\mathbf{t}_{\beta} \cdot \mathbf{t}_{\gamma}=\mathbf{t}_{\gamma} \cdot \mathbf{t}_{\alpha}=-\frac{1}{2} .
$$

This tensor is introduced as follows. In a reference configuration, select a domain $\Omega$ that is larger than distance between nodes, count all links that contain at least one node 
in $\Omega$, and call them a set $\Omega_{L}$. Set $\Omega_{L}$ is divided into three subsets $l_{\alpha}, l_{\beta}, l_{\gamma}$ of differently oriented links.

Now count the number $b_{\alpha}, b_{\beta}, b_{\gamma}$ of broken links in each family and introduce the relative directional damage,

$$
p_{\alpha}=\frac{b_{\alpha}}{l_{\alpha}}, \quad p_{\beta}=\frac{b_{\beta}}{l_{\beta}}, \quad p_{\gamma}=\frac{b_{\gamma}}{l_{\gamma}}, \quad p_{i} \in[0,1]
$$

Damage tensor $\mathbf{D}$ is introduced as a symmetric tensor:

$$
\mathbf{D}(\Omega)=p_{\alpha} \mathbf{t}_{\alpha} \otimes \mathbf{t}_{\alpha}+p_{\beta} \mathbf{t}_{\beta} \otimes \mathbf{t}_{\beta}+p_{\gamma} \mathbf{t}_{\gamma} \otimes \mathbf{t}_{\gamma} .
$$

Its eigenvalues and equal to

$$
\begin{aligned}
d_{1} & =\frac{1}{2}\left(p_{\alpha}+p_{\beta}+p_{\gamma}-\sqrt{p_{\alpha}^{2}+p_{\beta}^{2}+p_{\gamma}^{2}-p_{\alpha} p_{\beta}-p_{\beta} p_{\gamma}-p_{\alpha} p_{\gamma}}\right) \\
d_{2} & =\frac{1}{2}\left(p_{\alpha}+p_{\beta}+p_{\gamma}+\sqrt{p_{\alpha}^{2}+p_{\beta}^{2}+p_{\gamma}^{2}-p_{\alpha} p_{\beta}-p_{\beta} p_{\gamma}-p_{\alpha} p_{\gamma}}\right),
\end{aligned}
$$

One can see that the eigenvalues are nonnegative, $d_{1}$ is zero if only one family is damaged (crack-like directional damage) and $d_{2}$ is zero if and only if there is no damage at all, $p_{\alpha}=p_{\beta}=p_{\gamma}=0$. If all links are damaged, $p_{\alpha}=p_{\beta}=p_{\gamma}=1$, then $d_{1}=d_{2}=\frac{3}{2}$.

If the basis is chosen so that

$$
\mathbf{t}_{\alpha}=\left\{\begin{array}{l}
0 \\
1
\end{array}\right\}, \quad \mathbf{t}_{\beta}=\frac{1}{2}\left\{\begin{array}{c}
\sqrt{3} \\
1
\end{array}\right\}, \quad \mathbf{t}_{\gamma}=\frac{1}{2}\left\{\begin{array}{c}
\sqrt{3} \\
-1
\end{array}\right\},
$$

then the damage tensor assumes a form

$$
\mathbf{D}=\frac{1}{4}\left[\begin{array}{cc}
3 p_{\beta}+3 p_{\gamma} & \sqrt{3}\left(p_{\beta}-p_{\gamma}\right) \\
\sqrt{3}\left(p_{\beta}-p_{\gamma}\right) & 4 p_{\alpha}+p_{\beta}+p_{\gamma}
\end{array}\right] .
$$

Dimensionless damage tensor $\mathbf{D}$ allows the characterization of an average damage in a region $\Omega$ and hence the average irreversible deformation in that region. It can be used for an intermediate scale description of the damage. This tensor can be defined for all lattices, but in the bistable links, there are two damage tensors, one for the sacrificial elements and one for the waiting elements. For example, the damage tensor $\mathbf{D}$ shown in Fig. $3 \mathrm{~b}$ can be expressed as

$$
\mathbf{D}=\frac{1}{4}\left[\begin{array}{cc}
\frac{15}{16} & -\frac{\sqrt{3}}{16} \\
-\frac{\sqrt{3}}{16} & \frac{9}{16}
\end{array}\right], \quad \text { and } \quad d_{1,2}=\frac{3}{4} \pm \frac{\sqrt{3}}{8} .
$$

\subsection{Effectiveness Criterion based on the projectile}

An integral criterion that is not sensitive to the details of the damage is suggested by [7] where the variation of the impulse of the projectile is measured. It is assume that the projectile hits the structure flying into it vertically down. To evaluate the effectiveness, 
the ratio $R$ in the vertical component $\Lambda_{v}: \boldsymbol{\Lambda}_{p}=\left[\Lambda_{n}, \Lambda_{v}\right]^{T}$ of the impulse $\boldsymbol{\Lambda}_{p}$ of the projectile before and after the impact:

$$
R=\frac{\Lambda_{v}\left(T_{\text {final }}\right)}{\left|\Lambda_{v}\left(T_{0}\right)\right|}
$$

where $T_{0}$ and $T_{\text {final }}$ are the initial and the final moments of the observation, respectively. The variation of impulse of the projectile $R$ shows how much of it is transformed to the motion of structural elements. Parameter $R$ evaluates the structure's performance using the projectile as the measuring device without considering the energy dissipated in each

element of the structure; it does not vary when the projectile is not in contact with the structure.

\section{Elastic-brittle waiting elements}

Consider structural waiting elements that significantly increase the resistivity of the structure due to their morphology. These elements and their quasistatic behavior are described in [8]. Following [8], consider the link as an assembly of two elastic-brittle rods, lengths $L$ and $\Delta(\Delta>L)$ joined by their ends. The longer bar is initially slightly curved to fit. When an increasing external elongation stretches the link, only the shortest rod resists in the beginning. If the elongation exceeds the critical value, this rod breaks and the next (longer) rod then assumes the load replacing the broken one as previous shown in Fig. 2.

Assume that a unit amount of material is used for both rods. This amount is divided between the shorter and longer rod: The amount $\alpha$ is used for the shorter (first) rod and the amount $1-\alpha$ is used for the longer (second) one. The cross-sections $s_{1}$ and $s_{2}$ of rods are

$$
s_{1}(\alpha)=\frac{\alpha}{L} \quad \text { and } \quad s_{2}(\alpha)=\frac{1-\alpha}{\Delta} .
$$

The force-displacement relationship in the shorter rod is

$$
F_{1}(z)=k s_{1}(\alpha)\left(\frac{z}{L}-1\right)\left(1-c_{1}\right),
$$

where $c_{1}=c_{1}(z, t)$ is the damage parameter for this rod; it satisfies the following equation

$$
\frac{d c_{1}(z, t)}{d t}= \begin{cases}v_{d} & \text { if } z \geq z f_{1} \text { and } c_{1}(z, t)<1 \\ 0 & \text { otherwise }\end{cases}
$$

where $z f_{1}=L\left(1+\varepsilon_{f}\right), c_{1}(z, 0)=0$, and $v_{d}$ is the speed of damage. The longer rod starts to resist when the elongation $z$ is large enough to straighten this rod. After the rod is straight, the force-displacement relationship is similar to that for the shorter rod:

$$
F_{2}(z)=\left\{\begin{array}{ll}
k s_{2}(\alpha)\left(\frac{z}{\Delta}-1\right)\left(1-c_{2}\right) & \text { if } z \geq \Delta \\
0 & \text { if } z<\Delta
\end{array} .\right.
$$

Here $F_{2}$ is the resistance force and $c_{2}=c_{2}(z, t)$ is the damage parameter for the second rod: 


$$
\frac{d c_{2}(z, t)}{d t}= \begin{cases}v_{d} & \text { if } z \geq z f_{2} \text { and } c_{2}(z, t)<1 \\ 0 & \text { otherwise }\end{cases}
$$

where $c_{2}(z, 0)=0$. These equations are similar to Eqs. (11) and (12), where the crosssection $s_{1}(\alpha)$ is replaced by $s_{2}(\alpha)$ and the critical elongation $z f_{1}$ by $z f_{2}=\Delta\left(1+\varepsilon_{f}\right)$. The difference between the two rods is that the longer (slack) rod starts to resist only when the elongation is large enough. The total resistance force $F(z)$ in the waiting element is the sum of $F_{1}(z)$ and $F_{2}(z)$ :

$$
F(z)=F_{1}(z)+F_{2}(z)
$$

The graph of this force-displacement response for the monotonic external elongation is shown in Fig. 2 where the damage parameters jump from zero to one at the critical point $z f_{1}$.

\subsection{Kinematic of the waiting elements}

In the current research, the Finite Element Method (FEM) is chosen as the numerical tool. The numerical formulation can typically be founds in many FEM literatures, such as $[9,10]$. A brief overview of the technique is discussed in this section. The kinematic of the system can be described by the momentum equation

$$
\sigma_{i j, j}+\rho f_{i}=\rho \ddot{x}_{i},
$$

satisfying the following boundary conditions

- Traction boundary conditions

- Displacement boundary conditions

- Contact discontinuity

where $\sigma_{i j}$ is the Cauchy stress, $\rho$ is density, $f_{i}$ is the body force density, $\ddot{x}_{i}$ is acceleration, and $n_{j}$ is a unit normal to the element boundary. Using the principle of minimal virtual work $\pi$, the variation $\delta \pi$ can be expressed as

$$
\delta \pi=\int_{V} \rho \ddot{x}_{i} \delta x_{i} d V+\int_{V} \sigma_{i j} \delta x_{i, j} d V-\int_{V} \rho f_{i} \delta x_{i} d V-\int_{S} t_{i} \delta x_{i} d S=0 .
$$

The considered body is then discretized into finite elements defined by their nodal points

$$
\sum_{e=1}^{n}\left(\int_{V^{e}} \rho \ddot{x}_{i} \delta x_{i} d V+\int_{V^{e}} \sigma_{i j} \delta x_{i, j} d V-\int_{V^{e}} \rho f_{i} \delta x_{i} d V-\int_{S^{e}} t_{i} \delta x_{i} d S\right)=0
$$

Here, $n$ is the number of the nodal points defining element $e$. The variation $\delta x_{i}$, or the weight function, is defined as

$$
\delta x_{i}=w_{i}\left(X_{\alpha}, t\right)=N_{j}(X \alpha) w_{i}^{e}(t) \quad \text { and } x_{i}\left(X_{\alpha}, t\right)=N_{j}(X \alpha) x_{i}^{e}(t),
$$

where $N_{j}$ is the element shape function (or interpolating function), $x_{i}^{e}$ is the element nodal coordinate, and $w_{i}^{e}$ is the element weight function.

The model studied in this work is constructed using truss elements. The truss element carries an axial force and has three degree of freedom at each node. The displacement 
and velocity within the truss are interpolated in the local system $\hat{x}$ along the element's axis as follow [11]:

$$
u=N_{j} u_{i} \quad \text { and } \quad \dot{u}=N_{j} \dot{u}_{i},
$$

where $N_{1}=1-\hat{x} / L$ and $\hat{x} / L$, and $L$ is the element length.

\subsection{Constitutive model}

Consider a 1D-spar (truss) element, an elastic-brittle bistable behavior can be developed based on the formulation presented in the previous section. Stresses at time step $n+1$ are computed through stresses $\sigma^{n}$ of time step $n$

sacrificial element:

$$
\sigma_{11}^{n+1}=\left[\sigma_{11}^{n}+\frac{E_{s} \operatorname{tr} \Delta \varepsilon}{3(1-2 \nu)}+2 G_{s}\left(\Delta \varepsilon_{11}-\frac{1}{3} \operatorname{tr} \Delta \varepsilon\right)\right]\left(1-c_{1}^{n}\right),
$$

waiting element:

$$
\sigma_{11}^{n+1}=\left\{\begin{array}{ll}
{\left[\sigma_{11}^{n}+\frac{E_{w} \operatorname{tr} \Delta \varepsilon}{3(1-2 \nu)}+2 G_{w}\left(\Delta \varepsilon_{11}-\frac{1}{3} \operatorname{tr} \Delta \varepsilon\right)\right]\left(1-c_{2}^{n}\right)} & \text { if } c_{1}^{n} \geq 1 \\
0 & \text { if } c_{1}^{n}<1
\end{array},\right.
$$

where $\sigma_{11}$ is the axial stress, $\Delta \varepsilon$ is the strain increment tensor, $E$ is Young modulus, $\nu$ is Poisson's ratio, $G$ is the shear modulus, and $c_{1}$ is the damage parameter. The subscript $s$ and $w$ in moduli denote sacrificial and waiting element, respectively. The parameter $c_{i}$ controls the status of the links; $0=$ undamaged, $1=$ broken, and $0<c_{i}<1$ implies partially damaged. It also must satisfy the following

$$
c_{1}^{n}= \begin{cases}0 & \text { if } \varepsilon_{\text {total }} \leq \varepsilon_{c} \text { and } c_{1}^{n-1} \neq 1 \\ c_{1}^{n-1}+\dot{c}_{1} \Delta t & \text { if } \varepsilon_{\text {total }} \geq \varepsilon_{c} \text { and } c_{1}^{n-1}<1 \\ 1 & \text { otherwise }\end{cases}
$$

where $\varepsilon_{c}$ is the critical strain. When failure of the sacrificial element occurs, Eq. (21) sets stress to be zero, and the waiting element picks up the load after that as shown in Eq. (22).

\subsection{A two-dimensional assembly of the waiting elements}

Consider a unilateral triangular grid: each inner point has six equally distant neighbors, see Fig. 4. The distance between neighboring knots is equal to $L$. The knots in the boundary (including corners) have lower number of neighbors. The waiting elements connect the neighboring knots. Initially, the system is in equilibrium and does not have any inner stress. If the strain is small everywhere and each link is strained less than the critical value $z f$, the system is linear and it models a linear elastic material. After the first rod in a link breaks and is replaced with a longer one, the net experiences an "irreversible phase transition." 


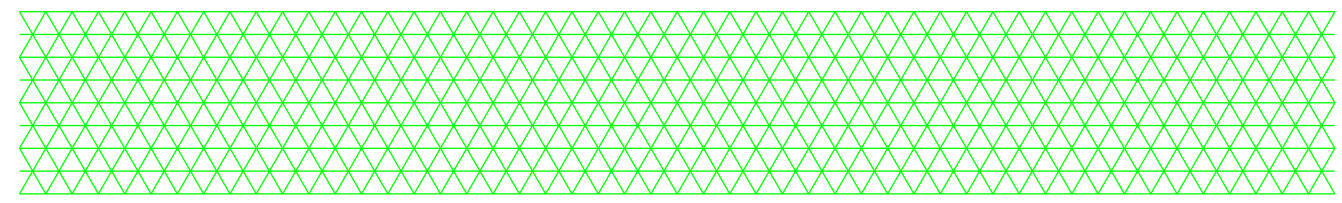

Fig. 4. The waiting element mechanism and its the force-displacement response

Table 1

sacrificial element's parameters

\begin{tabular}{ll} 
Parameter & Value \\
\hline Young modulus, $E$ & $120 \mathrm{GPa}$ \\
Poisson's ratio, $\nu_{12}$ & 0.36 \\
Length, $L$ & $1.0 \mathrm{~m}$ \\
Cross-sectional area, $A_{s} 0.01 \mathrm{~m}^{2}$ \\
Critical strain, $\varepsilon_{c}$ & 0.008 \\
\hline
\end{tabular}

Table 2

waiting element's parameters

\begin{tabular}{ll} 
Parameter & Value \\
\hline Young modulus, $E$ & $120 \mathrm{GPa}$ \\
Poisson's ratio, $\nu_{12}$ & 0.36 \\
Length, $L$ & $1.008 \mathrm{~m}$ \\
Cross-sectional area, $A_{s}$ & $0.0198 \mathrm{~m}^{2}$ \\
Critical strain (after fully stretched), $\varepsilon_{c}$ & 0.008
\end{tabular}

After each break, the network changes its elastic properties and its equilibrium position. The dynamics of the damage and failure of the net can be viewed as a series of these transitions. In contrast with the conventional structure, the waiting elements structure may become stronger after sacrificial elements break. Another advantage is that the additional slackness that is added after the break helps to spread the damage across the structure. A partially destroyed structure may have inner stresses.

\section{Numerical examples}

\section{Example 1: Single element test}

A single element test is performed to verify that the constitutive model is correctly implemented. The model parameters are given in Table 1 and 2 . The force-displacement relationship of a single-element test under uniaxial strain loading is shown in Fig. 6. Scale and rate effects are not considered, and all the parameters are tuned to the specific geometry chosen for this example in order to obtain the desired bistable response to be used in the next example.

Figure 5 illustrates that the amount of the energy dissipated also depends highly on the speed, $\dot{c}$, at which the link becomes fully damaged. The updated damaged parameter at each time step is computed using Eq. (23), hence, the size of the time step also greatly influences how fast the damage parameters changes from zero to one. For demonstration purposes, we have picked $\dot{c}$ to be $10^{6} \mathrm{~m} / \mathrm{s}$ for this particular example to mimic the elasticbrittle response and also to ensure that the sacrificial element is fully damaged before the waiting element becomes active. 


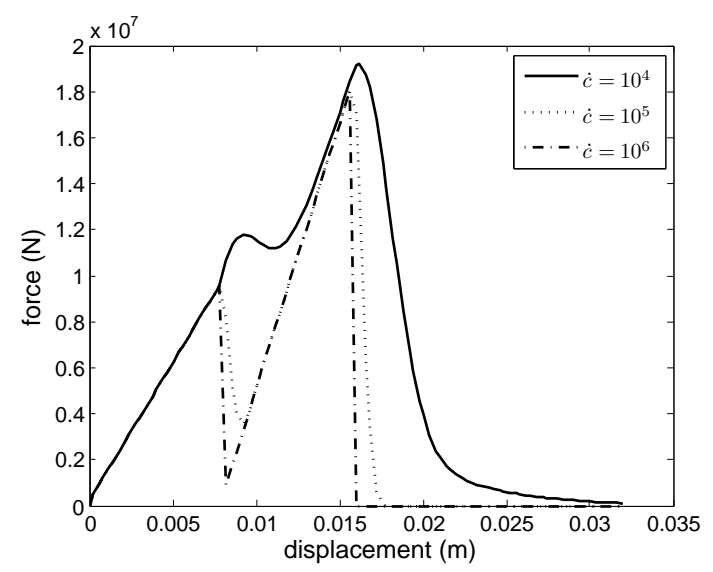

Fig. 5. Single element test: force-displacement diagram with various damage speed, $\dot{c}$

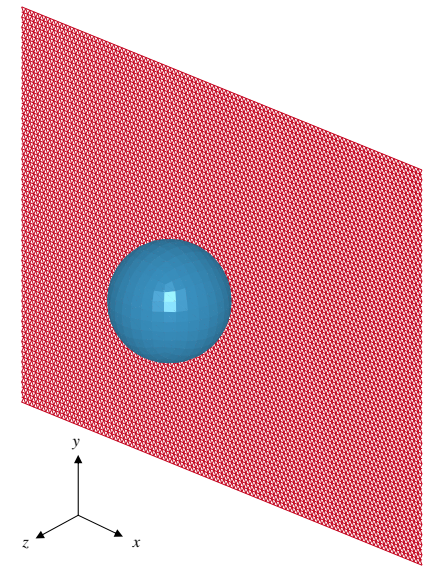

Fig. 6. Schematic sketch of the ball impacting net

\section{Example 2: A rigid ball impacting a net}

This example illustrates the performance of a bistable net-like structure under impact loading. Using the properties given in Table 1 and 2, a 100x100m net constructed with bistable links is impacted by a rigid body as shown in Fig. 6 . The net is constrained in all direction along the edge. The net is constructed using 23539 truss elements, and contains 8404 nodes. It should be noted that the parameters in this example are tuned for demonstration of concepts so that the differences of the outcome can significantly be seen between the net made from conventional links and bistable links.

The ball final velocity ( $t=0.5 \mathrm{~s}$ in this study), are given in Fig. 7. For the chosen configuration, at lower mass $(2500 \mathrm{~kg})$, the ball is rejected and neither type of the nets are damaged. As the mass is increased to $7500 \mathrm{~kg}$, the ball penetrates both nets and have similar exit velocity. The bistable net has the most advantage over the conventional net when the initial velocity of the ball is $150 \mathrm{~m} / \mathrm{s}$ and the mass of the ball is $5000 \mathrm{~kg}$. At this combination, the ball is bounced off the bistable net, while the conventional net is penetrated, see Fig. 8a. In addition, Fig. 8b shows that more energy is dissipated by the bistable net than the conventional net. These plots illustrate the range at which the the nets can effectively resist the impact.

The stress distribution comparison of the bistable net and the conventional net are shown in Fig. 9 and Fig. 10. In conventional net, the stress is uniformly and heterogeneously scattered when some neighboring links are destroyed. Similar pattern is observed for the bistable net once the waiting element are destroyed. However, when the sacrificial elements but not the waiting elements are destroyed, the stress distribution is more homogeneous. The conventional net is destroyed sooner than the one with waiting elements, and requires less energy to be penetrated.

The improved energy dissipation is achieved through the delocalization of damage. As can be seen from Fig. 11, damage in the bistable net is distributed throughout the net by completely destroying the sacrificial elements. The damage in the conventional net, on the other hand, concentrates in the impact area.

Another phenomenon observed from these simulations is the crack propagation. Illus- 


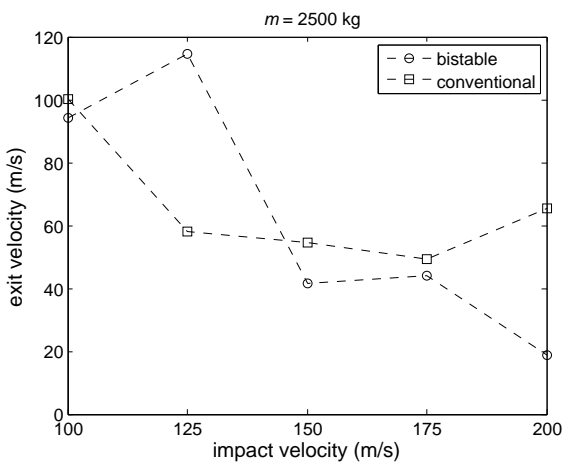

(a)

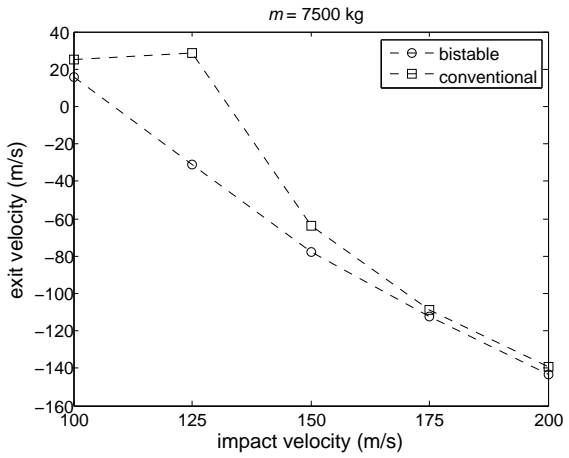

(c)

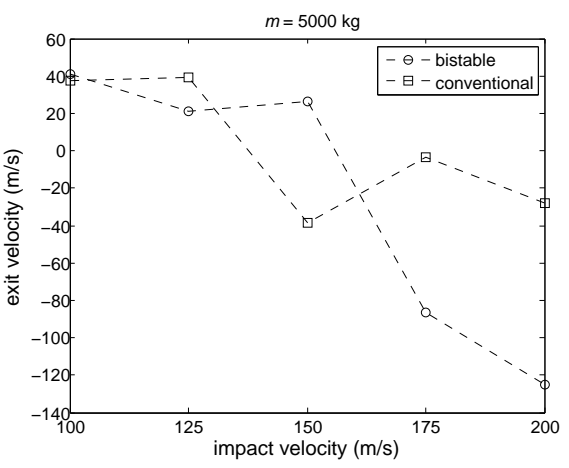

(b)

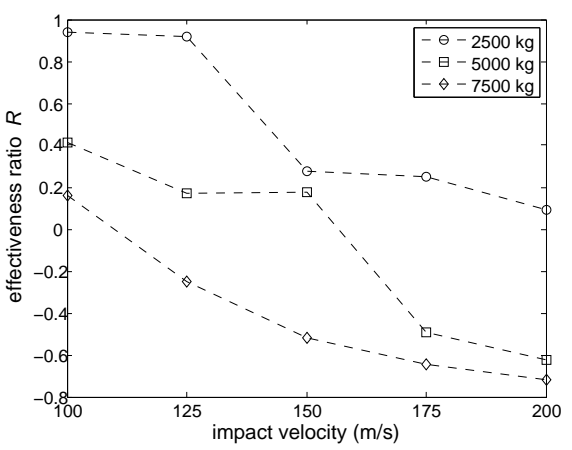

(d)

Fig. 7. (a)-(c) Comparison of the ball velocity at $t=0.5 \mathrm{~s}$. The positive velocity implies that the ball bounces off the net, while the negative velocity indicates that the ball continues moving in the direction that it is fired off. (d) Effectiveness ratio of the bistable net under an impact of various masses. The net is effective against impact when the $m \leq 5000 \mathrm{~kg}$ and $v_{\text {impact }} \leq 150 \mathrm{~m} / \mathrm{s}$.

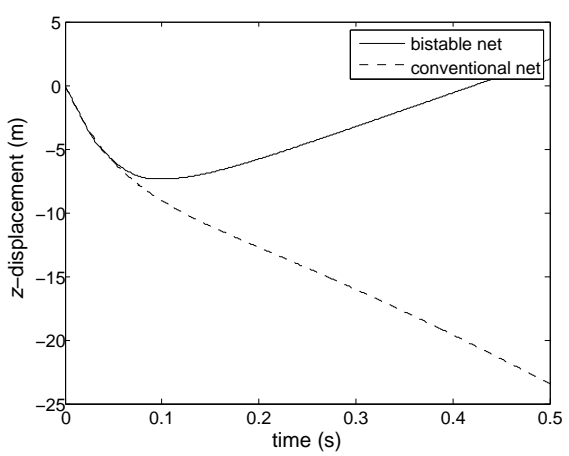

(a)

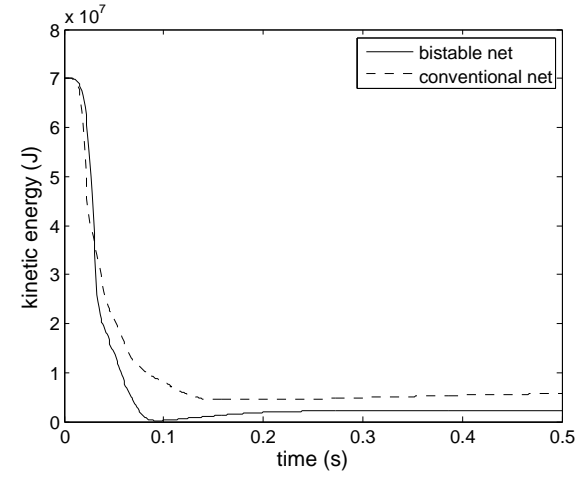

(b)

Fig. 8. (a) Kinetic energy. Mass $=5000 \mathrm{~kg}$ and impact velocity $=150 \mathrm{~m} / \mathrm{s}$. (b) Displacement along the $z$-direction; mass $=5000 \mathrm{~kg}$ and impact velocity $=150 \mathrm{~m} / \mathrm{s}$. 


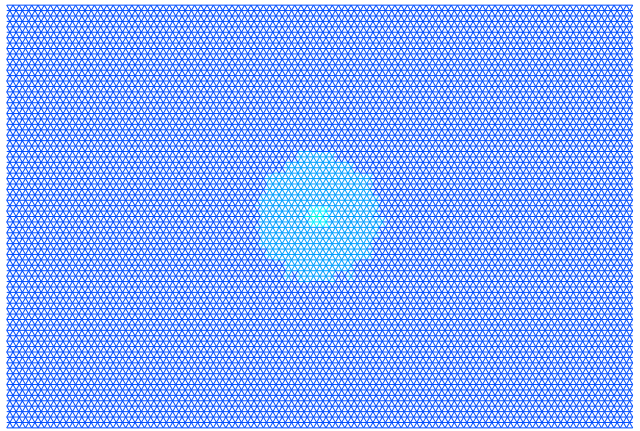

$t=0.014 \mathrm{~s}$

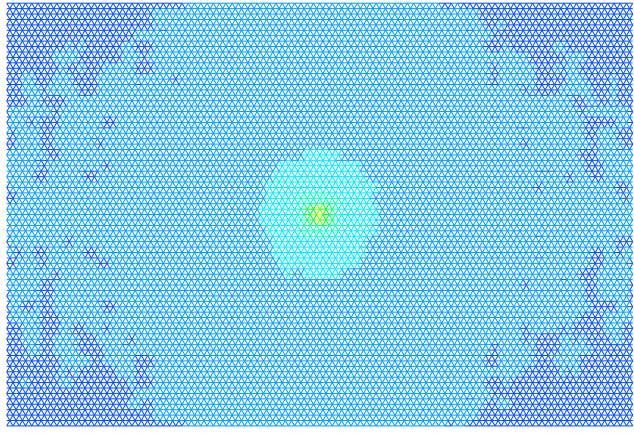

$t=0.022 \mathrm{~s}$

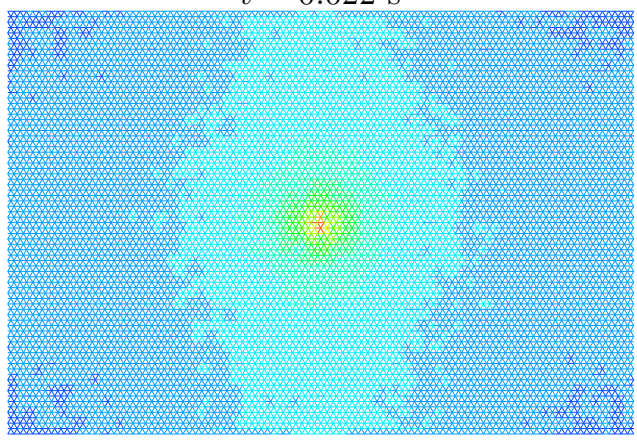

$t=0.030 \mathrm{~s}$

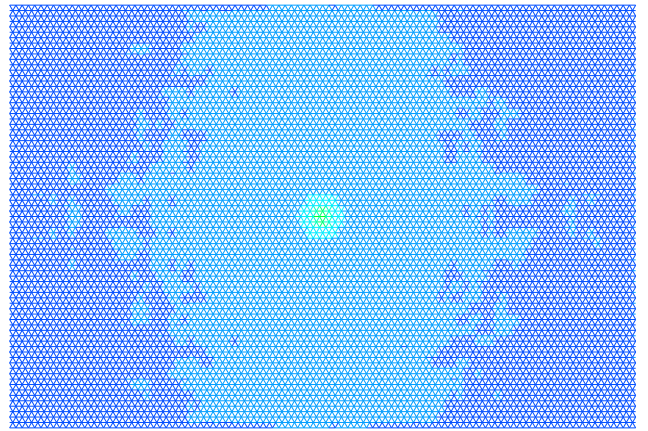

$t=0.019 \mathrm{~s}$

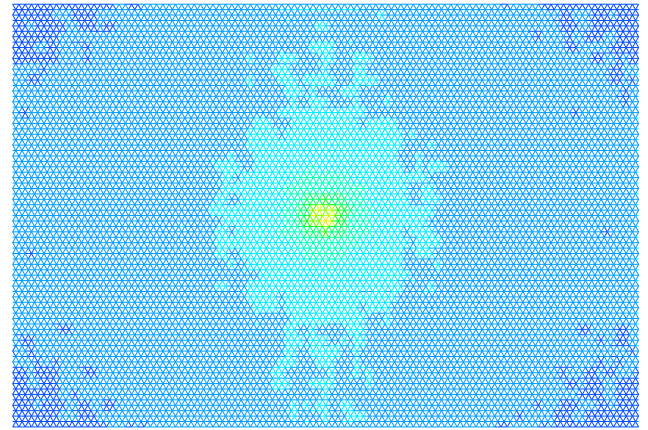

$t=0.026 \mathrm{~s}$

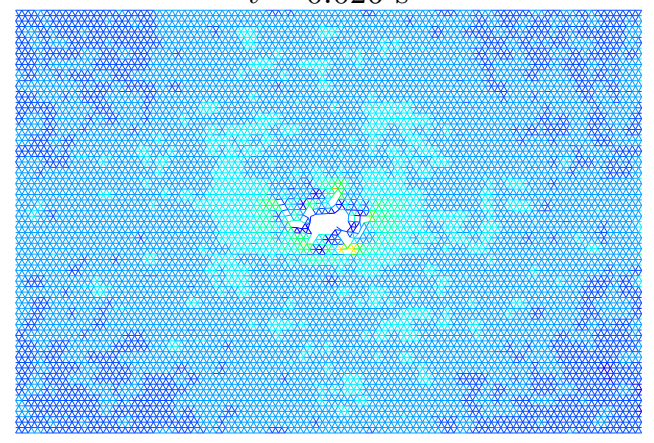

$t=0.034 \mathrm{~s}$

Fig. 9. Snapshots of axial stress in the net with bistable links

trated in Fig. 12, as the ball penetrates through the net, cracks are formed in the conventional net, while no crack formation is observed in the bistable net. Crack is stopped in the bistable because the delocalization via the waiting element. This illustrates that a waiting elements design prevents stress concentration that is the main cause of the construction failure, and therefore is preferable as a protective device. The bistable links, made from an elastic-brittle material, show the plastic-type behavior, see Fig. 2. Bistable links lead to large but stable pseudo-plastic strains and thus increase the resistivity. 


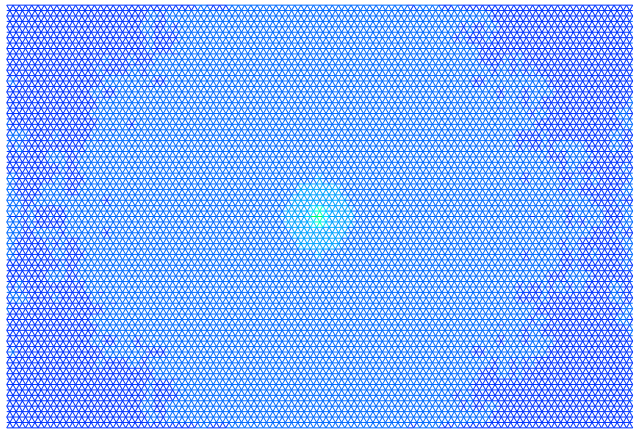

$t=0.014 \mathrm{~s}$

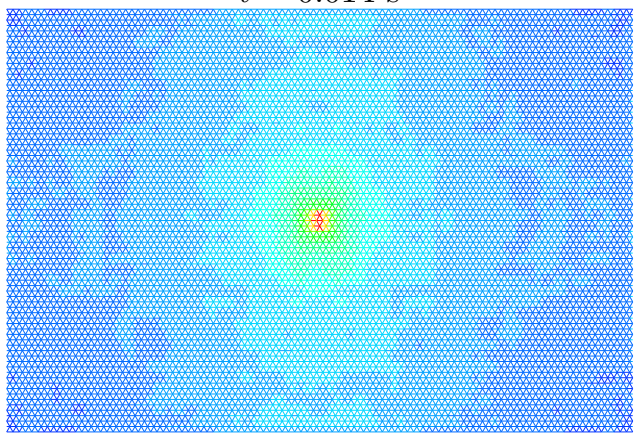

$t=0.022 \mathrm{~s}$

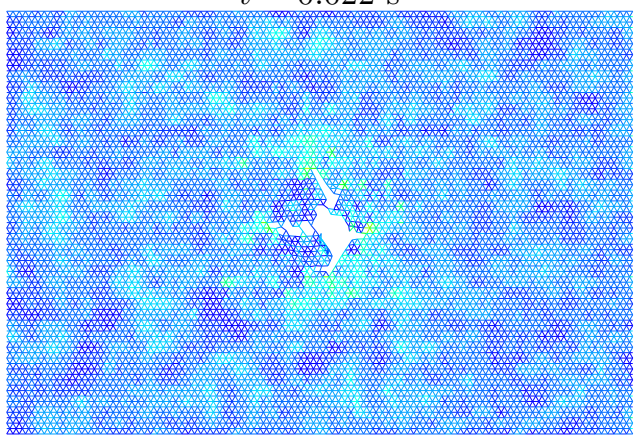

$t=0.030 \mathrm{~s}$

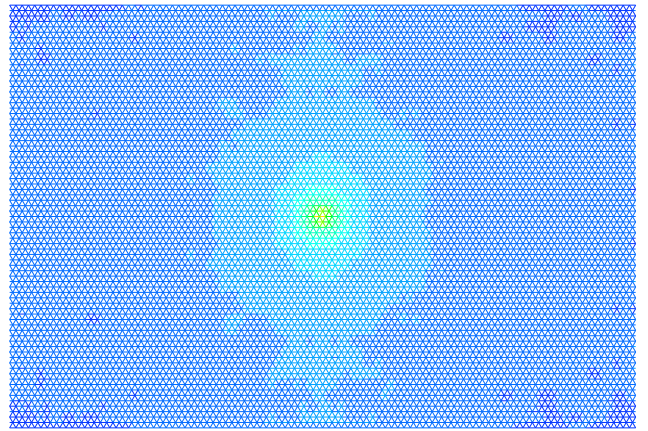

$t=0.018 \mathrm{~s}$

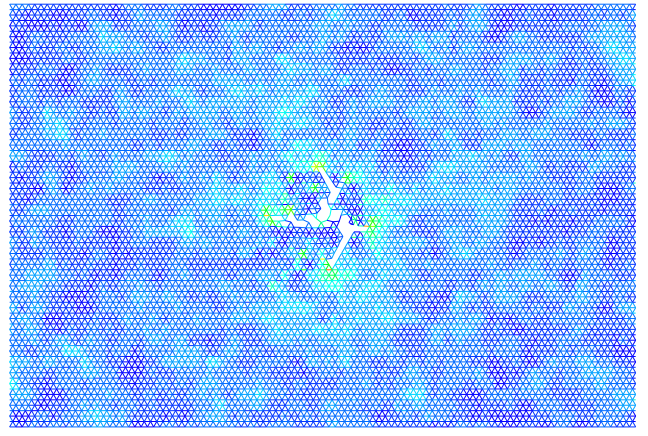

$t=0.026 \mathrm{~s}$

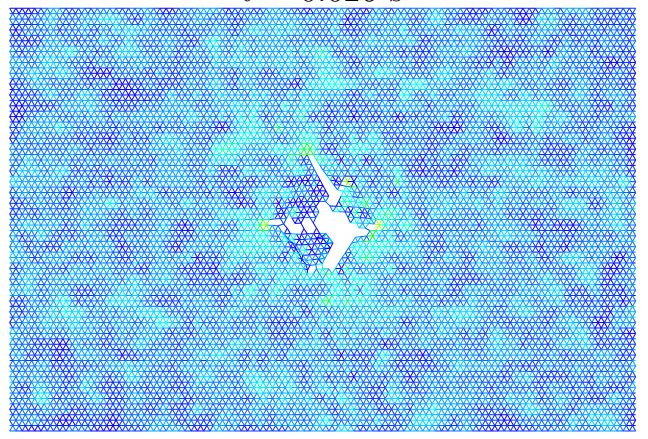

$t=0.034 \mathrm{~s}$

Fig. 10. Snapshots of axial stress in the net with regular links

\section{Conclusion}

The bistable structure can absorb more energy in comparison to the conventional structure by distributing the damage along its length. This behavior is due to special morphology of "waiting elements." The damage spread through created local instabilities in bistable structures can be implemented at all scales, from molecular to materials to large construction. The multiscale design forces more structural damage at each level and thus increases resistance of the structure. Correspondingly, the implementation is to be done by chemical and material design methods, by structural optimization and by 


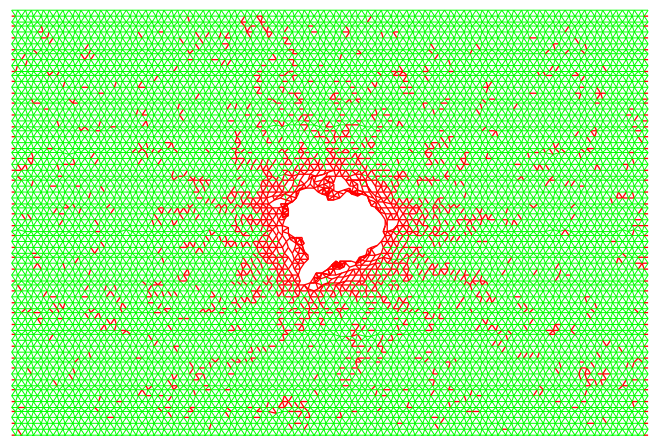

(a) Bistable net

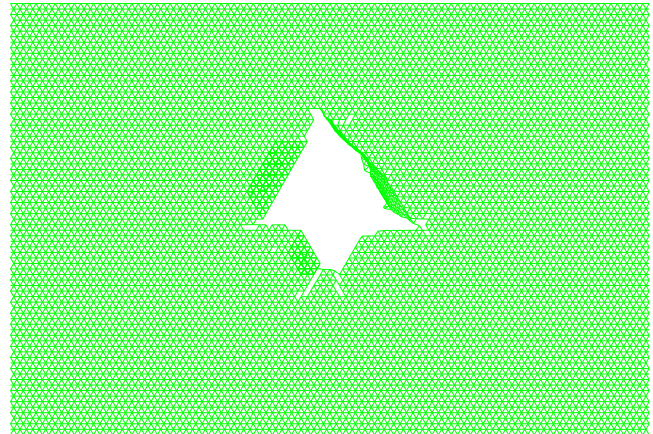

(b) Conventional net

Fig. 11. Damage at $t=0.5$ s.: (a) The complete destroyed sacrificial elements are shown in red (2435 damaged links), while the completely damaged waiting element are hidden from view. There are no partially damaged links in this example because the damage speed $\dot{c}$ is set to the same value as the time step size used in the simulation. (b) The completely destroyed links (284 links) are hidden from view.

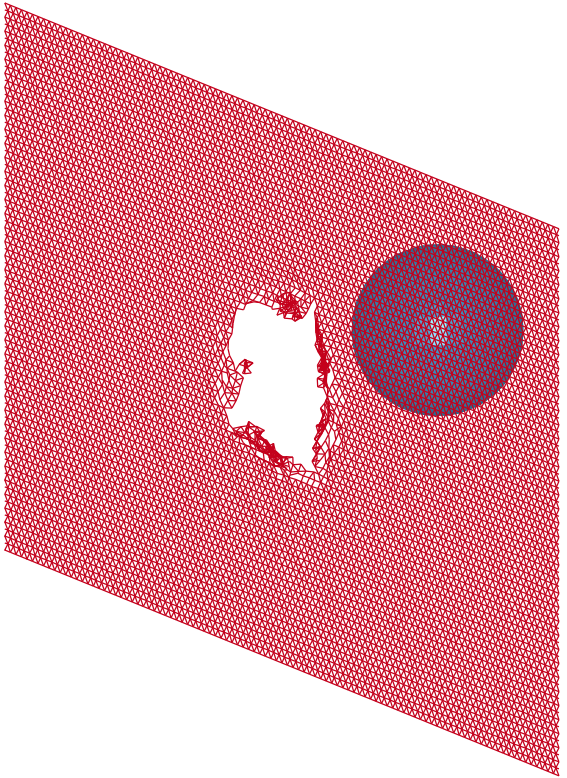

(a) Bistable net

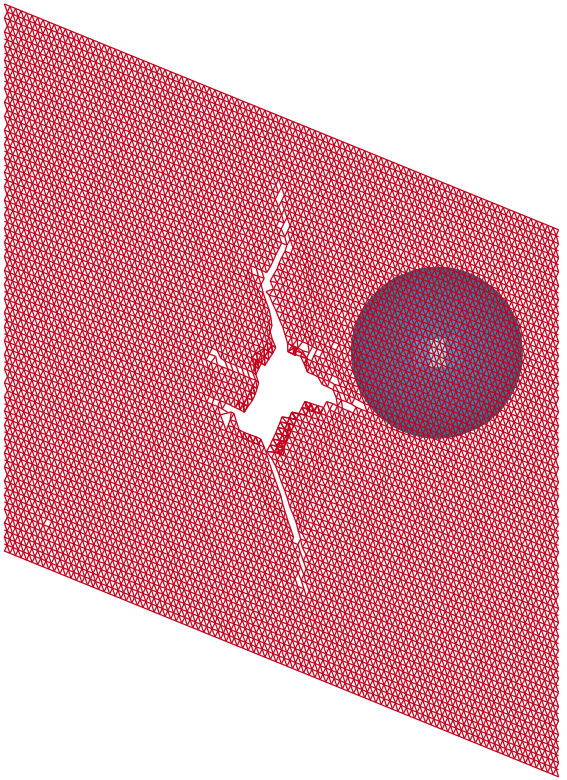

(b) Conventional net

Fig. 12. Penetration of a $7500 \mathrm{~kg}$ rigid ball with initial velocity of $175 \mathrm{~m} / \mathrm{s}, t=0.3 \mathrm{~s}$. The phase transition leads to a pseudo-plastic response of the net.

design principles that should be embedded into CAD system. The design of structures with bistable elements requires advanced numerical simulation and structural optimization, because the advantages of a bistable structure appear only if it is properly designed. The suggested bistable structures can protect against blasts and collisions. The immediate applications include helmets, safety nets, light weight armor, bunkers, bumpers and collision-protective devices for vessels. 


\section{Acknowledgements}

Support of this work by the Army Research Office (ARO) is gratefully acknowledged.

\section{References}

[1] T. Krauthammer, Modern Protective Structures, CRC Press, New York, 2008.

[2] A. V. Cherkaev, L. I. Slepyan, Waiting element structures and stability under extension, Journal of Applied Mechanics 4 (1) (1995) 58-82.

[3] L. Slepyan, M. Ayzenberg-Stepanenko, Localized transition waves in bistable-bond lattices, Journal of the mechanics and physics of solids 52 (7) (2004) 1447-1479.

[4] L. I. Slepyan, A. V. Cherkaev, E. Cherkaev, Transition waves in bistable structures. i. delocalization of damage, Journal of the mechanics and physics of solids 53 (2005) 383-405.

[5] L. I. Slepyan, A. V. Cherkaev, E. Cherkaev, Transition waves in bistable structures. ii. analytical solution: Wave speed and energy dissipation, Journal of the mechanics and physics of solids 53 (2005) 407-436.

[6] V. Vinogradov, A. V. Cherkaev, S. Leelavanichkul, The wave of damage in elastic-plastic lattices with waiting links: design and simulation, Mechanics of Materials 38 (2006) 748-756.

[7] A. V. Cherkaev, L. Zhornitskaya, Protective structures with waiting links and their damage evolution, Multibody System Dynamics 13 (1) (2004) 63 - 67.

[8] A. V. Cherkaev, L. Zhornitskaya, Dynamics of damage in two-dimensional structures with waiting links, Asymptotics, Singularities and Homogenisation in Problems of Mechanics (2004) 273-284.

[9] K. J. Bathe, Finite Element Procedures, Prentice Hall, New Jersey, 1996.

[10] R. D. Cook, D. S. Malkus, M. E. Plesha, R. J. Witt, Concept and Applications of Finite Element Analysis, 4th Edition, John Wiley \& Sons, Inc., New York, 1996.

[11] J. O. Hallquist, LS-DYNA Theory Manual, Livermore Software Technology Corporation, 7374 Las Pasitas Road, Livermore, CA (March 2006). 